\title{
The Limitations of Fair Division
}

\author{
AN EXPERIMENTAL EVALUATION OF THREE PROCEDURES
}

\author{
GERALD SCHNEIDER \\ ULRIKE SABRINA KRÄMER \\ Department of Politics and Management \\ Universität Konstanz, Germany
}

\begin{abstract}
Mathematical procedures that promise an envy-free, equitable, and efficient solution to distributional conflicts have received widespread attention. Two fair-division mechanisms, adjusted Knaster and proportional Knaster, which are similar to the well-known adjusted-winner procedure, are compared with the less fair divide-and-choose mechanism. Results show that participants largely prefer the adjusted-Knaster procedure to the two alternatives. Adjusted Knaster, closely followed by proportional Knaster, also promises the highest average payoff. Yet the sophisticated mechanisms cease to perform better than divide-and-choose once actors receive the possibility to deviate from the mandatory bargaining protocols of fair-division procedures. The preference for adjusted and proportional Knaster is found to be a partial function of the participants' psychological profile. The more "antisocial" a participant, the more likely this respondent is to opt for a procedure with a compensatory mechanism.
\end{abstract}

Keywords: fair division; experimental political science; bargaining; two-person games; proportional Knaster; adjusted winner; Knaster

$\mathbf{W}$ hen negotiating over Belfort after the Franco-German War, Chancellor Bismarck experienced an outburst from Foreign Minister Thiers. Reminding his counterpart that the disputed town never belonged to Germany, the French diplomat shouted,

You admit with your behavior that you have settled on a course of total destruction. Lead it! Demolish our provinces, burn our houses, strangle our innocent people! With one word: Finish your work. We will fight until the last breath. We will lose, but we will at least not be dishonored. (Quoted in Uthmann 1985, 178, authors' translation)

AUTHORS' NOTE: Previous versions of this article were presented at the annual convention of the International Studies Association, New Orleans, 2002; at seminars at Harvard University, the University of Kentucky, Universität Konstanz, and Europa-Universität Viadrina Frankfurt (Oder). The authors thank the Research Council of the University of Konstanz for financial support and Gabriele Ruoff for her able research assistance. We received helpful comments from Steven Brams, Thomas Bräuninger, Oliver Fabel, Matt Gabel, Werner Güth, Matthias Raith, David Wildasin, and Sonja Ziniel. The replication data set and the questionnaire (both the original German version and a translation) can be downloaded from the replication homepage of the first author: http://www.uni-konstanz.de/FuF/Verwiss/GSchneider/. The data used for this study are also available at http://www.yale.edu/unsy/jcr/jcrdata.htm/.

Konstanzer Online-Publikations-System (KOPS)

URL: http://www.ub.uni-konstanz.de/kops/volltexte/2007/2402/

URN: http://nbn-resolving.de/urn:nbn:de:bsz:352-opus-24028 
The immediate consequence of the heated exchange was that Belfort ultimately remained French. Yet as we sadly know from the history of the 20th century, the lack of magnanimity that Bismarck showed in the negotiations fueled the tensions between the two states to such an extent that further militarized confrontations became even more likely.

The episode illustrates the difficulties negotiators have in slicing up contested territories. The scientific study of international politics confirms that striking a deal that all participants consider acceptable is especially difficult in rivalries (Diehl and Goertz 2000) and in international mediation of disputes involving incompatible negotiation positions (Bercovitch and Langley 1993). Obviously, fair division problems abound elsewhere, too. Many divorcees remain frustrated over the way courts divide property, rule on child custody, and assign spousal and children support duties (Emery 1999). In business, "fairness" is often invoked in disputes over the question of whether firms should have a right on the "value" of their employees after a contract expires. The infamous Bosman ruling of the European Court of Justice exemplifies these difficulties. The abolishment of transfer payments gave soccer players more power but left those clubs that invest heavily in the training of young talents deeply dissatisfied. ${ }^{1}$

The ubiquitous complexities in finding a solution that is acceptable to all stakeholders involved in a distributive dispute have motivated game theorists and mathematicians to develop fair-division procedures. Especially the solutions presented in two monographs by Brams and Taylor $(1996,1999)$ have revived the quest for a "magic formula" to resolve conflicts. Based on the pioneering work by Knaster (1946), Steinhaus (1948), and others, Brams and Taylor propose two procedures-adjusted winner and proportional allocation - that ought to ensure "fair outcomes;" that is, an outcome everybody agrees upon and characterizes as fair. Both mechanisms are built on the premise that opponents who receive the more valuable share of indivisible goods will compensate the less fortunate party in the negotiation process. As Brams and Taylor amply demonstrate, these two procedures offer several advantages over conventional fair-division procedures that families and other social groups use to share goods.

This article uses an experimental research design to test the applicability of fairdivision procedures. We evaluate in particular whether two procedures-proportional Knaster (PK) and adjusted Knaster (AK), a slight modification of adjusted winner that is easier to implement (Raith 2000) - yield better results than procedures we know from daily experience. Our empirical evaluation employs the divide-and-choose (DC) procedure as a benchmark of comparison. To make them applicable in an experimental setting, we have slightly transformed the AK and PK procedures. We also evaluate which one of the two sophisticated mechanisms entices more truthful behavior from the negotiation partners.

Adding to the growing experimental literature on fairness (e.g., Fehr and Schmidt 1999), our article demonstrates that the applicability of these mathematical procedures is limited. Sophisticated fair-division mechanisms prove to be advantageous

1. The ruling has its name from Belgian soccer player, Jean-Marc Bosman, who was unable to transfer freely from R.C. Liège to a French club, US Dunkerque, when his contract expired in 1990. 
only in situations where the negotiators are forced to strictly live up to the demands of the fair-division protocol and where there is no possibility to renegotiate the contract after a first agreement. Once the bargaining protocol that the disputants impose upon themselves by agreeing on a fair-division procedure ceases to be binding, "primitive" procedures, such as DC, perform like the more complicated PK and AK procedures. If actors have, for example, the chance to change their valuation of the contested goods or to withhold compensation to the opponent, the collective yield of the two sophisticated mechanisms is considerably reduced. Our multivariate models also reveal quite disconcertingly that the preference for a procedure not only depends on the expected utility calculations of the negotiators but also on their psychological profile.

The remainder of this article is structured as follows: We first introduce the literature on fair division and present the essential features of the three procedures under examination. Next, we present the experimental design and some summary statistics about the 119 participants in our experiments. The empirical evaluation starts with the examination of whether the two sophisticated procedures are preferred to the benchmark mechanism, DC, and whether they yield higher and fairer gains. We then move to a multivariate analysis of the psychological and procedural underpinnings of the participants' choices. The article concludes with practical and theoretical recommendations.

\section{A COMPARISON OF DIFFERENT FAIR-DIVISION PROCEDURES}

Fair-division procedure is a catchall term for bargaining protocols that help negotiators in achieving mutually acceptable negotiation outcomes. An outcome is considered to be fair if it fulfills certain criteria. In their seminal works on this issue, Brams and Taylor (1996, 1999) list five requirements: (1) proportionality, (2) envy-freeness, (3) equitability, (4) efficiency, and (5) truthfulness. We can postulate as a rule of thumb that the more criteria a fair-division procedure meets, the fairer it is. To live up to all demands simultaneously becomes more difficult with a growing number of players with similar preferences (Edelman and Fishburn 2001). We limit our evaluation, however, to interactions between two persons.

1. Proportionality: The contentment with a bargaining outcome depends on the degree to which each party receives a portion according to the relative importance of an actor. If the parties are equal, the proportionality criterion implies that each of them has the impression to have gained at least half of the total value.

2. Envy-freeness means that each party believes that she or he possesses the largest or most valuable part of the bargain. In other words, an envy-free participant would not want to trade her or his share against the portion the other negotiator receives. ${ }^{2}$ In two-party situations, envy-free outcomes are necessarily proportional.

2. Brams and Taylor $(1996,241)$ introduce a subjective element into their definition of envy-freeness and write, "An allocation is envy-free if every player thinks he or she receives a portion that is at least tied for largest, or tied for most valuable and, hence, does not envy any other player." 
3. Equitability is an equally subjective criterion and expresses the conviction of the two partners that they received the same fraction of the spoils. ${ }^{3}$ The concept of equitability requires individuals to engage in interpersonal utility comparisons. Although orthodox economics dismisses models based on this notion, there is ample empirical evidence showing that equitability and envy-freeness are important considerations in human decision making.

4. Efficiency equals the well-known Pareto criterion, meaning that any alternative to the achieved deal would hurt the interests of some participant. Note that efficiency alone does not guarantee a fair solution because a $90 \%$ to $10 \%$ division would also be efficient.

5. We also introduce the criterion of truthfulness or sincerity to which Brams and Taylor (1996) pay less attention than to the other evaluation standards. Truthfulness, a benchmark derived from noncooperative game theory, means that participants express their preferences sincerely; that is, the preference orders they make manifest in their claims reveal their real preference orders. A sincere individual follows her or his preference order also in cases where the chosen strategy is not part of a Nash equilibrium. ${ }^{4}$ In the practical context of our experiment, this solution concept particularly requires that a person does not change her or his course of action in case she or he obtains privileged information or the possibility to withhold a compensatory payment.

One could add further criteria to which a fair-division procedure should live up in real-word negotiations. Just as the axiomatic bargaining models of the 1950s and 1960s, such mechanisms follow the logic of cooperative game theory. Allowing us to deduce optimal solutions under a binding bargaining protocol, they constitute normative rather than positive models of political choice (Raith 2000). The implicit "rules of the game" include, for instance, the assumptions that the bargaining process is limited to one round, that the negotiations are costless, and that renegotiations are impossible. If we were to relax such assumptions, we would have to invoke noncooperative solution concepts, such as "renegotiation proofness" (e.g., Farrell and Maskin 1989) to evaluate a particular solution.

However, this article does not explore whether existing fair-division procedures meet the demands of additional criteria. We pursue a much more modest research strategy and assess how some of the recently proposed procedures perform in an experimental setting. In other words, we try to determine if these widely discussed mathematical innovations meet the expectations of their inventors. Even though the normative models under consideration represent idealized bargaining contexts, we believe it necessary to confront them with data to test whether they are applicable at all. Up to now, empirical work on fair-division procedures is fairly anecdotal or restricted to single cases (e.g., Massoud 2000).

The thrust of our empirical examination is similar to the studies by Frohlich and Oppenheimer (1992) and Ostrom, Gardner, and Walker (1994). Frohlich and Oppenheimer evaluate Rawls's (1971/1999) work on distributive justice experimentally, in particular showing that agents do not necessarily apply the minimax criterion

3. Brams and Taylor $(1996,241)$ define it as follows: "An allocation is equitable for two players if each player thinks that the portion he or she receives is worth the same, in terms of his or her valuation, as the portion that the other player receives in terms of that player's valuation."

4. The Nash equilibrium is an outcome where none of the actors has an unilateral incentive to choose an alternative. 
favored in A Theory of Justice to choose social arrangements. ${ }^{5}$ Ostrom, Gardner, and Walker analyze how different institutional and informational setups affect the contribution to a common pool resource. They buttress their main finding-namely, that collaboration does not break down in the absence of strong enforcement mechanismswith impressive quasi-experimental and experimental evidence.

Following the logic of these experimental institutional analyses, our work will focus on three fair-division procedures. The first procedure, which will provide a null model, is the well-known DC mechanism. According to this straightforward negotiation protocol, the first person divides the goods, whereas the second one has the right to choose a portion. The divider receives the remaining share. Obviously, DC is easy to implement. In our experiment, the participants assigned to this procedure had to divide money and indivisible goods. We examine only the choices made by the divider. In a bilateral setting, DC can lead to an efficient, fair, and equitable outcome, although this is not guaranteed. The dividing person might, for instance, split the goods strategically and separate the objects in a way that she or he receives the most preferred object and all the money. In our setup, we provided this incentive by informing the divider about how much the other person valued the goods. To exploit the other side in this way is, however, not a bulletproof strategy, because the actor with the first move advantage has no possibility to exactly know how the chooser values the money compared to the goods.

The name of the second procedure, adjusted Knaster (AK), refers to the Polish mathematician Bronislaw Knaster (1946) who made pioneering contributions to the literature on fair division. The adjective adjusted relates to the adjusted-winner mechanism developed by Brams and Taylor $(1996,1999)$. Raith (2000) introduces the AK procedure and extensively discusses its merits. In his view, it marries "the efficient adjustment method of the Knaster procedure with the equitability condition of adjusted winner" (p. 308). In addition, its compensation mechanism is much easier to implement compared to the adjusted-winner procedure.

AK and other advanced fair-division procedures confront participants in a first step with a sealed-bid auction over the contested goods. Accordingly, we asked participants to allocate 100 points among three goods: a ballpoint pen, a lighter, and a mug, each with the price of 5 German Marks. Assume that the first participant's valuation $\{6,67,27\}$ placed high priority on the lighter, but the second negotiator $\{5,34,61\}$ preferred the mug. In this case, each participant receives the good she or he valuated higher than her or his counterpart. Thus, the first actor receives the lighter and the ball pen and therefore reaches 73 points. The other person gets the mug and 61 points. The second phase of the procedures involves a straightforward compensation scheme. The first person compensates her or his counterpart monetarily according to her excess points, which amount to 12 . Consequently, the second actor would receive a monetary compensation that equals 6 points.

The second of the more sophisticated mechanisms we evaluate is PK. It initially follows the same auction scheme as AK. For this purpose, however, we modify the "pro-

5. A recent study by Michelbach et al. (2003) rehabilitates the Rawls conjecture. 
portional-allocation" procedure that is extensively discussed by Brams and Taylor (1996). According to them, proportional allocation encourages more truthful behavior but also entails less efficient results than adjusted winner. ${ }^{6}$ The practical problem of this procedure is, however, the relative difficulty to calculate payoffs. To render the procedure applicable in dividing indivisible goods, we use a simplified proportionality scheme for the compensatory mechanism. PK and AK exclusively differ in the last stage of the interaction. We asked participants again to allocate 100 points among three objects. If the two valuations of the three goods are $\{6,67,27\}$ and $\{5,34,61\}$, under the PK procedure each participant has to give half of the monetary value of the goods he or she has received to his or her opponent. That is, participant 1 compensates participant 2 with half of the material value of the 1st and 2nd good, participant 2 in turn compensates participant 1 with half of the material value of good 3. Although the PK mechanism consequently deals with the monetary and therefore a relatively objective value of the goods, the compensation scheme of AK requires more subjective reasoning.

Because the advantages of $\mathrm{PK}$ and $\mathrm{AK}$ are obvious, we would expect that most participants would prefer these mechanisms over DC. Furthermore, the application of the sophisticated procedures should guarantee efficient and equitable outcomes. Our empirical examination inquires whether these mechanisms live up to these expectations. We evaluate in particular to what extent the two procedures with a compensatory scheme, AK and PK, fall victim to strategic behavior once the bargaining protocol is no longer binding. We analyze how vulnerable a procedure is by counting the number of people who change their valuation after receiving information on the valuation of the other actor or by calculating how much compensation was withheld if the participants were given this option. ${ }^{7}$

We also examine if individual characteristics influence the type of procedure a participant chooses and the size of the payoff. In particular, we explore whether the attitudes of the participants have an impact in this regard. Although we could potentially choose from a plethora of sociopsychological scales, we employ only measures that constitute proxies for rational choice concepts: dominance, social responsibility, antisocial attitude, and security need. ${ }^{8}$ We constructed the scales in accordance with the coding rules listed in Schneewind and Graf (1998) and Hathaway, McKinley, and Engel (2000).

According to the sociopsychological literature, the first concept, dominance, refers to the tendency of imposing one's own will on other people (Schneewind and Graf 1998). Dominant people try to control the behavior of others. Strongly dominant individuals often care little about others, whereas their subservient and obedient counterparts push their own emotions aside and take the wishes of others into account. In case

6. Brams and Taylor $(1996,78)$ conclude the comparative assessment of proportional allocation and adjusted winner by saying "that PA induces truthfulness, at least to a high degree, but it is purchased at the cost of the efficiency that AW (adjusted winner) gives."

7. Our experimental setting is thus comparable to a divorce where, in the beginning, a couple bargained according to a fair-division procedure but where one actor deviates from the socially optimal outcome. This can be done by keeping back alimonies.

8. A more detailed rational as to why we relied on these variables and not other ones can be found in Krämer and Schneider (2003). 
of conflict, they are likely to give in for the sake of peace or to avoid further confrontation. Dominance implies the willingness to exploit first move advantages and similar opportunities. The dominance scale measure uses seven categories, ranging from subservient to strongly dominant (Schneewind and Graf 1998). ${ }^{9}$ In the construction of this variable, the raw data were transformed in accordance with the general coding rules to account for age- and sex-specific norms.

Social responsibility, by contrast, measures how much an actor takes the well-being of other persons into account. People who score high on this scale attribute the consequences of their behavior to themselves and consider themselves both reliable and trustworthy. They also like to take over responsibility within their own group. Participants with a low value, conversely, do not easily take responsibility for their own actions. They seldom believe themselves to be reliable and trustworthy, attribute low levels of integrity to themselves, and do not feel responsible for other people. The social responsibility scale uses three categories and is based on the Minnesota Multiphasic Personality Inventory-2 (MMPI-2) (Hathaway, McKinley, and Engel 2000).

Antisocial attitude similarly measures the degree of egoism an individual exhibits in a social interaction. Theoretically, the measure captures a different behavioral dimension than the social responsibility scale. Participants who score high on the former scale express beliefs that violate key norms of social life. Such respondents show a low respect for the law and see little value in an ordered social community. One cannot expect that such people care for the needs of others. The antisocial attitudes scale extends to three categories. This scale also stems from the MMPI-2 (Hathaway, McKinley, and Engel 2000).

The need for security, finally, expresses the extent to which an individual is riskaverse or risk-taking. Participants who score high on this scale regard social norms as important. They will be anticipatory in their behavior and try to assemble goods and values without risk-if possible, under a well-known institution. The scale on the need for security is taken from the Character-Structure-Test (CST) inventory (Sponsel 1982). This six-category measure relates to the need for material and immaterial security. The higher a value, the more people are concerned with security and are strongly aware of social values and the importance of norms. ${ }^{10}$

\section{EXPERIMENTAL DESIGN AND METHOD}

The experiment was conducted at the University of Konstanz, Germany. We used handbills and posters to recruit 119 subjects to participate in the fair-division experiment. All sessions were held in the academic year 2001-02. The experiment fell into different phases, roughly summarized as introduction, selection, and division. After having introduced the experiment to the participants, we presented the general rules of

9. We also ran the statistical models with scales in which the number of categories was reduced. The results did not fundamentally change.

10. Although the antisocial attitude scale seems to correspond closely to the social responsibility scale, Spearman's rho for measuring the association between the two ordinal variables does not exceed -.32 
the three fair-division procedures. The instructor distributed the questionnaire and answered questions of the participants if necessary.

At this time, we also suggested to the individual participant who was sitting alone in a classroom that a fictitious partner "sits" in a neighboring room. ${ }^{11}$ One half of the participants were told that the interaction might be repeated with this pretended counterpart. We said to the other half that they would interact only once with their coplayer. Note that the reliance on fictitious actors is a common, although not completely unproblematic, practice in experimental research (Frohlich, Oppenheimer, and Moore 2001). The questionnaire required the participants to respond to 82 attitudinal questions, which we used afterwards to construct the behavioral scales.

The participants had to familiarize themselves with the three procedures in the second phase. We also asked some control questions to figure out whether they understood the procedures. Once this was done, they had to choose a division procedure. We asked them to allocate 100 points to the three procedures according to their preferences and told them that the fictitious second person would do the same. The questionnaire also states that the teams would afterwards go through the procedure for which they collectively garnered the highest number of points. Note again that the coplayer was fictitious and that only the point allocation of the real players affected the decision on the procedure that would be chosen for the last phase of the experiment. Furthermore, because we needed a comparable number of participants for each procedure, we had to assign some participants to different procedure from the one they originally opted for in the second phase. In the end, 52 test persons could participate in the division phase under the procedure of choice, whereas the rest had to be allocated to another mechanism. We will examine statistically whether this treatment had an effect on the size of the payoff that the average participant was able to obtain.

The participants were confronted with the three goods at the beginning of the division phase. All participants had the chance to see the valuation of their fictitious interaction partner. Half of the participants were asked to write down their own valuation before they saw the preferences of their counterpart. They could then decide whether to change the valuation. The other half, by contrast, were immediately allowed to see the valuation of the other side. We controlled in this fashion for the possibility that test persons shy away from changing their allocation when they have already made an initial choice. If it was necessary to compensate the other actor (following the rules of AK or PK), we gave participants the possibility to abstain from compensating. Yet the participants did not know beforehand that such an opportunity could arise. Although the logic of compensation schemes necessarily differs, we tried to make them compatible. A participant was entitled to compensation if she or he received fewer points (AK) or goods (PK) than her or his counterpart. They accordingly could count on 0.1 German Marks for each point of difference under AK and 2.50 German Marks for each good under PK.

We also gathered summary information on the background of the participants. In the statistical analysis, we employ five control variables to account for the demo-

11. The reliance on a fictitious coplayer allows us to control some ancillary conditions. Because test persons who often take place in experiments might see through this dodge, we also controlled for experimental experience in the statistical tests. 
graphic profile of our participants: $\operatorname{sex}($ female $=0$, male $=1$ ), age, social science student (no $=0$, yes $=1$ ), humanities student (no $=0$, yes $=1$ ), and previous experience with experiments (no $=0$, one $=1$, more than one $=2$ ).

Based on this information, we could establish that the average participant was 24.2 years old and in the seventh term of her or his studies. Thirty-seven participants participated for the first time and 21 for the second time in an experiment. Sixty-one students had already gained experience from more than one experiment before taking part in our behavioral tests of the fair-division procedures. There were 60 male and 59 female among the 119 participants. Thirty-one students were enrolled in the natural sciences (biology, chemistry, physics, math, statistics, and psychology), 63 in the social sciences (law, political science, management, economics, sociology, educational science, sports, and history), and 25 in the humanities (philosophy, art and media, literature, and languages). ${ }^{12}$

The participants' responses to our 82 attitudinal statements differ considerably. The four scales we used to test the influence of a participant's background on the experimental choices are all additive indices. We reduced the scales to ordinal measures with varying numbers of categories. ${ }^{13}$

Multivariate, multinomial logit models are the means to explore what kind of factor makes a difference in the choice of a fair-division procedure. Ordinary least squares (OLS) regressions help us to determine whether the procedure or the personal attributes of a respondent affect the size of the payoffs that were finally received. The first part of the empirical examination, to which we will turn next, is a more descriptive comparison of how the three fair-division procedures perform.

\section{THE PERFORMANCE OF THREE PROCEDURES}

In this section, we examine whether the performance of the three fair-division procedures differs when relying on the criteria of envy-freeness, equitability, efficiency, and truthfulness. The recent upsurge in the study of fair-division procedures led to the expectation that the more advanced mechanisms should outperform simplistic bargaining devices, such as DC, if two individuals have to split up indivisible goods and a limited sum of money.

Table 1 summarizes the comparative evaluation of the three procedures. We generally distinguish two forms of performance. Type I performance refers to the outcome that would have materialized in the event the test persons had not received incentives to deviate from their original valuation or to withhold compensation. Because we intro-

12. The figures do not necessarily add up to 119 because of missing values. The University of Konstanz uses a nontraditional faculty structure for which we corrected when we attributed a student to a general field of study. Although sociology is, for instance, affiliated with the historians at Konstanz, sociology students were coded as social scientists in our examination.

13. The questionnaire also included some statements that we used to categorize participants according to their tendency to lie. We used the L scale, which is a validation instrument, to establish whether a participant was not answering the questions in a sincere manner. Only 3 individuals scored relatively high on this measure, and excluding them from the analysis did not affect the results significantly. See the Minnesota Multiphasic Personality Inventory-2 (MMPI-2) for coding details (Hathaway, McKinley, and Engel 2000). 
TABLE 1

The Performance of Three Fair-Division Procedures

\begin{tabular}{|c|c|c|c|c|}
\hline & $\begin{array}{l}\text { Divide-and- } \\
\text { Choose }\end{array}$ & $\begin{array}{c}\text { Adjusted } \\
\text { Knaster }\end{array}$ & $\begin{array}{l}\text { Proportional } \\
\text { Knaster }\end{array}$ & $\begin{array}{c}\text { All } \\
\text { Procedures }\end{array}$ \\
\hline \multicolumn{5}{|l|}{ Preferences and payoffs } \\
\hline First choice $^{\mathrm{a}}$ & 30 & 64 & 14 & 108 \\
\hline Times applied & 40 & 38 & 41 & 119 \\
\hline Average valuation & $\begin{array}{l}27.00 \\
(26.09)\end{array}$ & $\begin{array}{l}49.23 \\
(31.05)\end{array}$ & $\begin{array}{l}23.85 \\
(20.55)\end{array}$ & $\begin{array}{l}33.36 \\
(28.13)\end{array}$ \\
\hline Average payoff in German Marks I & n.a. & $\begin{array}{c}17.32 \\
(3.21) \\
\{49.49 \%\}\end{array}$ & $\begin{array}{c}17.5 \\
(0.00) \\
\{50.0 \%\}\end{array}$ & $\begin{array}{l}17.4 \\
(2.34)\end{array}$ \\
\hline Average payoff in German Marks II & $\begin{array}{c}13.21 \\
(2.74) \\
\{52.84 \%\}\end{array}$ & $\begin{array}{c}20.11 \\
(3.36) \\
\{57.46 \%\}\end{array}$ & $\begin{array}{c}17.89 \\
(1.69) \\
\{51.11 \%\}\end{array}$ & $\begin{array}{l}18.59 \\
(2.81)\end{array}$ \\
\hline \multicolumn{5}{|l|}{ Equitability } \\
\hline Average difference in points $\mathrm{I}^{\mathrm{b}}$ & n.a. & $\begin{array}{c}17.83 \% \\
(12.01) \\
\{n=20\}\end{array}$ & $\begin{array}{c}15.29 \% \\
(12.37) \\
\{n=19\}\end{array}$ & $\begin{array}{c}16.59 \% \\
(12.09) \\
\{n=39\}\end{array}$ \\
\hline Average difference in points II ${ }^{\mathrm{b}}$ & $\begin{array}{l}13.41 \% \\
(13.81)\end{array}$ & $\begin{array}{l}21.53 \% \\
(11.15)\end{array}$ & $\begin{array}{l}21.49 \% \\
(12.03)\end{array}$ & $\begin{array}{l}18.76 \% \\
(12.88)\end{array}$ \\
\hline Average difference in German Marks I & n.a. & $\begin{array}{c}6.22 \\
(5.82) \\
\{n=20\}\end{array}$ & $\begin{array}{c}0.00 \\
(0.00) \\
\{n=19\}\end{array}$ & $\begin{array}{c}3.19 \\
(5.19) \\
\{n=39\}\end{array}$ \\
\hline Average difference in German Marks II & $\begin{array}{c}4.18 \\
(3.77)\end{array}$ & $\begin{array}{c}7.97 \\
(2.79)\end{array}$ & $\begin{array}{c}1.67 \\
(3.05)\end{array}$ & $\begin{array}{c}4.52 \\
(4.12)\end{array}$ \\
\hline Higher payoff of outcomes I & n.a. & $\begin{array}{l}35.00 \% \\
\{n=20\}\end{array}$ & $\begin{array}{r}0.00 \% \\
\{n=19\}\end{array}$ & $\begin{array}{l}17.95 \% \\
\{n=39\}\end{array}$ \\
\hline Higher payoff of outcomes II & $77.50 \%$ & $78.95 \%$ & $24.39 \%$ & $59.66 \%$ \\
\hline Equal payoff of outcomes I & n.a. & $\begin{array}{l}25.00 \% \\
\{n=20\}\end{array}$ & $\begin{array}{l}100.00 \% \\
\{n=19\}\end{array}$ & $\begin{array}{l}61.54 \% \\
\{n=39\}\end{array}$ \\
\hline Equal payoff of outcomes II & $0.00 \%$ & $0.00 \%$ & $70.73 \%$ & $24.37 \%$ \\
\hline \multicolumn{5}{|l|}{ Envy-freeness } \\
\hline $\begin{array}{l}\text { Cases in which both actors got at least } \\
50 \% \text { I }\end{array}$ & n.a. & $\begin{array}{c}75 \% \\
\{n=20\}\end{array}$ & $\begin{array}{c}68.42 \% \\
\{n=19\}\end{array}$ & $\begin{array}{c}71.79 \% \\
\{n=39\}\end{array}$ \\
\hline $\begin{array}{l}\text { Cases in which both actors got at least } \\
50 \% \text { II }\end{array}$ & \multicolumn{3}{|c|}{ Cases in which both actors got at least } & $15.97 \%$ \\
\hline \multicolumn{5}{|l|}{ Efficiency } \\
\hline Sum of points reached $\mathrm{I}^{\mathrm{b}}$ & n.a. & $\begin{array}{c}69.88 \\
(12.23) \\
\{n=20\}\end{array}$ & $\begin{array}{c}70.39 \\
(13.04) \\
\{n=19\}\end{array}$ & $\begin{array}{c}70.13 \\
(12.46) \\
\{n=39\}\end{array}$ \\
\hline Sum of points reached II $^{\mathrm{b}}$ & $\begin{array}{l}53.86 \\
(16.77)\end{array}$ & $\begin{array}{l}53.82 \\
(5.13)\end{array}$ & $\begin{array}{c}59.53 \\
(14.37)\end{array}$ & $\begin{array}{c}55.77 \\
(13.35)\end{array}$ \\
\hline
\end{tabular}


TABLE 1 (continued)

\begin{tabular}{|c|c|c|c|c|}
\hline & $\begin{array}{c}\text { Divide-and- } \\
\text { Choose }\end{array}$ & $\begin{array}{c}\text { Adjusted } \\
\text { Knaster }\end{array}$ & $\begin{array}{c}\text { Proportional } \\
\text { Knaster }\end{array}$ & $\begin{array}{c}\text { All } \\
\text { Procedures }\end{array}$ \\
\hline \multicolumn{5}{|l|}{ Truthfulness } \\
\hline Change in preferences ${ }^{c}$ & $\begin{array}{c}32 \% \\
\{n=40\}\end{array}$ & $\begin{array}{c}85 \% \\
\{n=20\}\end{array}$ & $\begin{array}{c}74 \% \\
\{n=19\}\end{array}$ & $\begin{array}{c}55.52 \% \\
\{n=79\}\end{array}$ \\
\hline Cases where no compensation paid & n.a. & $\begin{array}{c}67 \% \\
\{n=18\}\end{array}$ & $\begin{array}{c}33 \% \\
\{n=30\}\end{array}$ & $\begin{array}{l}45,83 \% \\
\{n=48\}\end{array}$ \\
\hline Average withheld compensation in DM & n.a. & $\begin{array}{c}6.27 \\
(2.41)\end{array}$ & $\begin{array}{c}2.5 \\
(0.00)\end{array}$ & $\begin{array}{c}4.55 \\
(2.59)\end{array}$ \\
\hline Average points from Nash equilibrium ${ }^{\mathrm{d}}$ & n.a. & $\begin{array}{c}\{n=12\} \\
22.3 \\
\{n=20\}\end{array}$ & $\begin{array}{c}\{n=10\} \\
65.3 \\
\{n=19\}\end{array}$ & $\begin{array}{c}\{n=22\} \\
43.23 \\
\{n=39\}\end{array}$ \\
\hline
\end{tabular}

NOTE: Standard deviations appear in parentheses. n.a. = nonapplicable.

a. Ten participants (5.04\% of total sample) claimed to be indifferent between two procedures. Each time 4 were indifferent between adjusted Knaster and proportional Knaster and between adjusted Knaster and divide-and-choose, 2 were indifferent between proportional Knaster and divide-and-choose. Information is missing for 1 participant.

b. Entries are standardized values because the potential points differ across the procedures.

c. The valuation change took for divide-and-choose the form that we counted the number of times that an individual obtained the good that he or she favored the most in the original valuation.

d. The Nash value of each object was calculated and compared to the number of points attributed to an object. The three differences were added.

duced such incentives for most interactions, the figures reported represent counterfactual estimates that would have been achieved in a world that forces persons to adhere to the bargaining protocol. Type II performance, by contrast, represents what the participants achieved in the real interactions.

We begin by evaluating how popular the fair-division mechanisms are. Table 1 presents counts of how often a procedure was the first choice among our participants. ${ }^{14}$ Another popularity measure is the average number of points a procedure received after the presentation of the procedures. We also calculated how much money the participants earned, including a uniform price of 5 German Marks for all goods in the bargain.

The relative number of times the actors realized or would have realized the same subjective outcome as the imaginary counterpart indicates the degree of equitability of a procedure. Envy-freeness is measured by counting the number of cases both actors, the participant and the fictitious second person, reached at least $50 \%$ of the monetary outcome. The efficiency measures calculate how many points the actors reached together. Obviously, a solution lies either in the Pareto set or not. Because it was not possible to lose anything in the experiments, our participants always agreed on an effi-

14. Recall that this frequency does not correspond to the number of times participants went through a certain division procedure. 
cient outcome. To distinguish different levels of success, we calculated the sum of the points reached for both actors.

Finally, the truthfulness criteria establish how often actors changed their behavior after receiving information about the other actor. We differentiate between changes made in the valuation and the percentage of cases in which participants withheld compensation. Further indicators are the average size of the unpaid compensations and the deviation of the outcome in points from the Nash equilibrium.

It becomes quickly apparent from Table 1 that AK is by far the most popular of the three mechanisms. The frequency in which AK was the preferred procedure is not too surprising because it relies on a more straightforward compensatory scheme than PK. In the beginning, $\mathrm{PK}$ and $\mathrm{AK}$ are the same procedures, but the former relies on a more complicated compensation scheme than the latter. The most preferred procedure also yielded the highest average monetary payoff in situations with incentives to deviate from the cooperative course. If we were looking only at what would have happened in a purely cooperative world, PK would fare marginally better.

It is rather surprising that approximately one of four participants preferred DC over the other mechanisms. We believe that many people opted for DC because they already knew this procedure. Admittedly, the relative popularity could also be partly due to the experimental situation. Participants could anticipate that their payoff would be only marginally lower than the one they would potentially obtain under the other procedures.

The first concept that we use to "evaluate" the fairness of a procedure is the criterion of envy-freeness. According to the literature, all three procedures should fulfill this requirement (Raith 2000; Brams and Taylor 1996, 1999). In situation I, in which actors have no unilateral incentives and no possibility to cheat, both AK, with $75 \%$, and PK, with $68.4 \%$, score relatively high. In situation II, in which actors receive the possibility to refuse compensatory payments, the figures change enormously. Only $18.4 \%$ of AK divisions and $29.27 \%$ of PK divisions are envy-free because they promised a higher or equal payoff to the one reached by the interaction partner. It is remarkable that DC leads in no single case to an envy-free result.

PK fares especially well on the criterion of equitability, where the number of cases in which both persons end up with exactly the same payoff is much higher than for the two other procedures. All cases of PK divisions would have met the criterion of equitability in situation I. But even in situation II, PK performs very well with $70.73 \%$ of equitable cases. A further indication of the good performance of $\mathrm{PK}$ is that the difference in the payoffs is the smallest for PK. AK and DC do not achieve one single equitable result in situation II.

If we allow participants to deviate from the fair-division protocol, DC performs considerably better. It has more outcomes in which the participants did not change their preferences than AK. The latter procedure, however, led to the highest average payoff in situations where the participants possessed the chance to change their valuations. This is a first indication that strategic behavior is particularly prominent under this procedure. It is not surprising that the efficiency of AK is strongly reduced if we allow for strategic interaction. The same is true for PK, although the reduction is less dramatic. If we compare the efficiency of PK and AK in situations without unilateral 
incentives, almost no difference exists. These results clearly indicate that the success of fair-division procedures crucially hinges on whether a bargaining protocol is binding. If actors are given the chance to behave strategically, the advantage of the sophisticated mechanism disappears.

Differences between AK and PK are manifest when we turn our attention to the truthfulness criterion. Only $33 \%$ of all participants who had a chance to do so withheld compensation, whereas the corresponding figure under AK amounts to $67 \%$. One reason for the relative high incidence of uncooperative behavior probably stems from the fact that the compensations are more difficult to calculate under PK than under AK. The amount of compensation withheld is on average smaller under the latter than under the former mechanism.

We assumed for the calculation of the Nash equilibria that an actor would typically try to receive as many objects as possible. If this person were after the division of objects in a position where she or he should compensate the other side, she or he would strive to minimize this compensation. In the contrary event, she or he should try to obtain as large a compensation as possible. The average sum of the differences between the Nash valuations and the ones actually chosen blurs that the variance across participants is considerable. The results also show that participants are not necessarily Nash optimizers when they are given the chance to behave strategically. Hence, individuals often use proxies when calculating their utility, although a great number of actors came quite close to the Nash optimum under AK.

In sum, the evaluation of the procedures shows that sophisticated mechanisms do not perform very well in situations where participants have the possibility to disregard the bargaining protocol. If actors are not able to exploit such incentives, fair-division works at the average. Because the precommitment to a cooperative course of action is not credible in some important contexts, which type of participant has a tendency to opt for a specific fair-division procedure must be examined. We will move to this question in the next section.

\section{THE SOCIAL PSYCHOLOGY OF FAIR DIVISION}

Although the two procedures with a compensatory scheme promise a higher level of influence and therefore a better outcome than the DC mechanism, approximately one of four participants would have preferred the customary bargaining protocol. Furthermore, although the average gain of $\mathrm{AK}$ and $\mathrm{PK}$ is highest, some participants who used these procedures did not fare better than the ones who had to rely on DC. This section explores whether psychological factors can account for these differences. While controlling for the possible impact of the participants' personal background, we explore the explanatory power of attitudinal factors on the preference for a certain procedure and also on the payoff they finally attained. As mentioned before, the four sociopsychological variables are dominance, responsibility, antisocial, and security. The control variables are the participants' sex, their age, whether they are enrolled in the social sciences or humanities, and finally whether they had already participated in social experiments. 


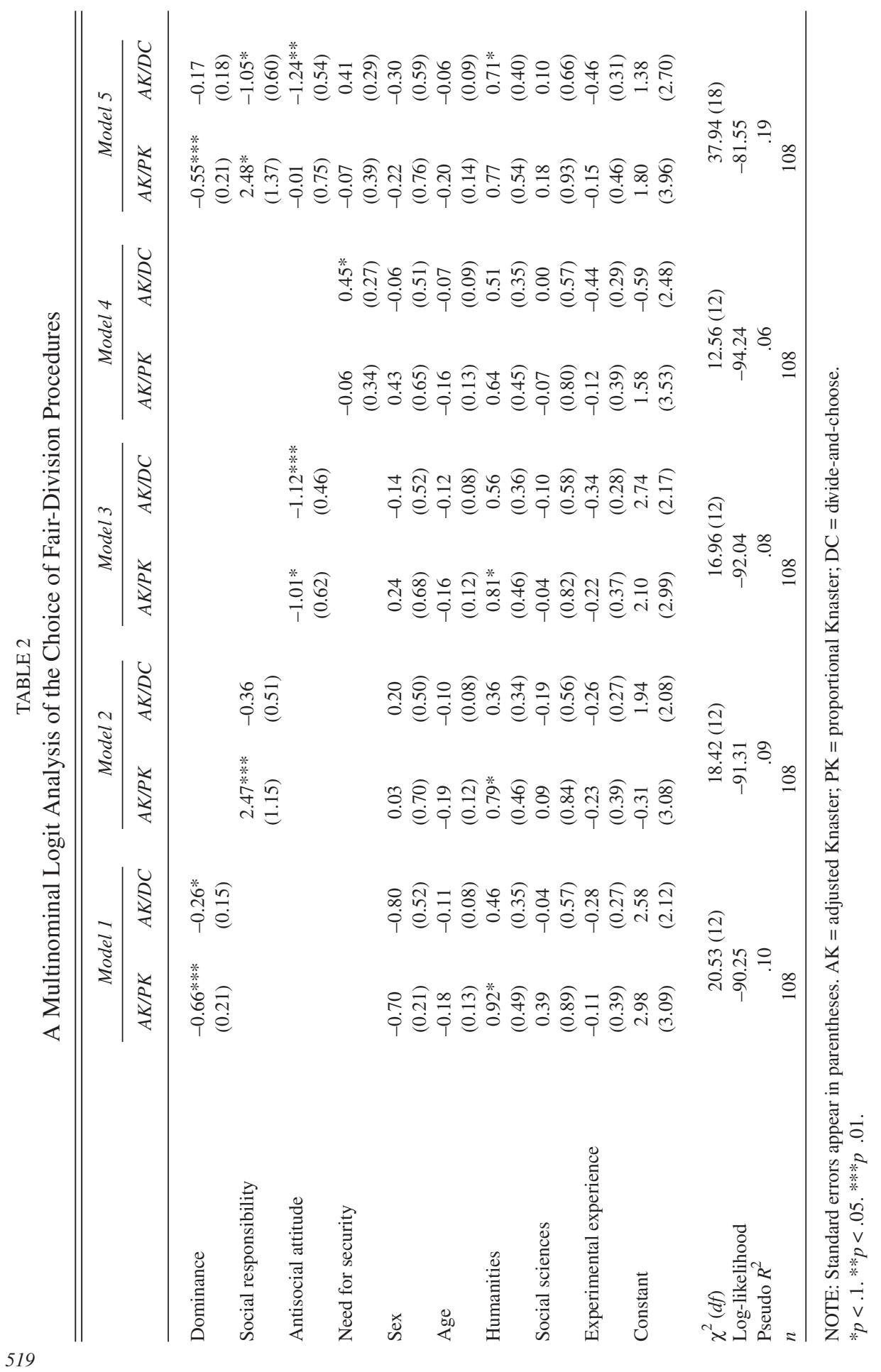


Table 2 first presents the results of five multinomial logit models that seek to explain the choice participants made in the selection phase of the experiment when we confronted them with the three procedures. Note again that participants did not yet know at this stage of the experiment what kind of good they could opt for in the division part of the experiment. Models 1 to 4 examine the impact of the individual psychological factors, and model 5 offers an estimation of the full model. Note that collinearity between the explanatory variables does not pose major problems. ${ }^{15}$

The results reported in Table 2 clearly show that psychological factors influence the procedural choices the individuals made in the selection phase of fair division. The more dominant a person is, the higher the likelihood that she or he will opt for AK. If we move from the lowest category to the highest one on this variable and leave the other explanatory variables at their means, the probability change of a participant's opting for this procedure amounts to .38 . We observe, conversely, that the likelihood that the other procedures are selected is a drop of .09 (DC) and .29 (PK). Individuals who seek security are, in contrast, much more prone to opt for DC. A move from the lowest to the highest category, again holding all the other variables at their mean values, increases the probability of this choice with a .28 change. The most surprising effect is arguably that people with an antisocial attitude are more likely to opt for AK than for DC. We can observe a probability change of .37 in this respect. The effects of a maximum change in social responsibility are similar. Again, holding all the other variables at their means, we observe a drop in the likelihood to opt for AK instead of DC amounting to .26 .

Gender differences, which frequently occur in experiments, do not become manifest in the institutional choices our participants made. Students in humanities are more likely to opt for DC, whereas an enrollment in social sciences does not exert a major effect. The sociopsychological variables have, however, a stronger influence than these control variables.

The final analytical step in our evaluation is to figure out whether psychological factors affect the payoffs in the division phase. We would like to test in the remainder of the article whether psychological variables have an impact on the final payoff that the participants received or whether procedural variables possess some explanatory power here. To this end, we include four procedural variables. Assigned procedure examines whether the mechanism at work has an impact on the results. The variable is coded 1 if the procedure was AK, 2 in case of PK, and 3 in event of the DC mechanism. The results did not change if we use two dummy variables instead of this tripartite indicator, which allows us to differentiate between mechanisms with and without compensatory scheme. Correspondence with chosen procedure measures whether a participant was assigned the procedure for which she or he had opted earlier in the experiment. We code the variable as 1 if there is a discrepancy between the chosen and the assigned procedure and 0 otherwise. The dichotomous concept number of rounds measures whether a participant receives information on a possible repetition of the

15. The Spearman correlation coefficients measuring the association between the ordinally scaled independent variables do not exceed 0.4 . Because multicollinearity can inflate the standard errors, we also conducted variance inflation tests. The corresponding values for the individual predictors are smaller than 1.5 . 
TABLE 3

The Impact of Psychological and Procedural Variables on the Payoffs of the Participants (ordinary least squares regressions)

\begin{tabular}{lccc}
\hline \hline & Model 1 & Model 2 & Model 3 \\
\hline Dominance & $-0.28(1.76)$ & & $1.50(1.52)$ \\
Social responsibility & $5.26(5.86)$ & & $-0.59(5.09)$ \\
Antisocial attitude & $4.60(5.33)$ & & $-0.21(4.59)$ \\
Need for security & $-1.30(2.77)$ & & $-3.77(2.41)$ \\
Assigned procedure & & $-15.93^{* * *}(2.77)$ & $-16.78^{* * *}(2.88)$ \\
Correspondence with chosen procedure & & $-12.25^{* * *}(4.75)$ & $-11.66^{* *}(4.78)$ \\
Number of rounds & & $11.63 * * *(4.54)$ & $12.36^{* * *(4.61)}$ \\
Timing of commitment & & $5.57(4.45)$ & $6.71(4.52)$ \\
Sex & $0.14(5.73)$ & $0.15(4.69)$ & $2.54(4.99)$ \\
Age & $1.12^{*}(0.66)$ & $0.58(0.55)$ & $0.35(0.57)$ \\
Humanities & $1.53(3.93)$ & $4.93(3.25)$ & $3.61(3.40)$ \\
Social sciences & $-0.44(6.48)$ & $-0.76(5.43)$ & $-2.25(5.60)$ \\
Experimental experience & $3.26(3.08)$ & $2.71(2.60)$ & $3.83(2.72)$ \\
Constant & $18.70(24.28)$ & $59.88 * * *(18.58)$ & $72.29 * * *(23.03)$ \\
& & & \\
$n$ & 119 & 119 & 119 \\
Prob. $>F$ & .61 & .00 & .00 \\
$F(d f)$ & $0.81(9,109)$ & $5.97(9,109)$ & $4.38(13,105)$ \\
Adjusted $R^{2}$ & -.01 & .27 & .27 \\
$R^{2}$ & .06 & .33 & .35 \\
\hline
\end{tabular}

$* p<.1 . * * p<.05 . * * * p .01$.

division. We announced before the interaction started whether there are eventually more rounds (coded as 0 ) or only one round (coded as 1 ). The final procedural variable is called timing of commitment. It measures whether the participants had first written down their valuation and then seen the valuation of their partner $(=1)$ or whether they had seen the valuation of their partner first before they committed themselves to a valuation $(=0)$. We will use a linear regression approach to test whether these procedural factors or the psychological background of the participants is more important for the payoff size. The analysis distinguishes between three statistical models. Model 1 takes the influence of the psychological variables into account, model 2 does the same for the procedural ones, and model 3 combines the two sets of predictors. All models include the controls that we already used at an earlier stage.

The results reported in Table 3 demonstrate clearly that only the procedural variables possess some predictive power for the payoff variance. Neither the controls nor the psychological concepts are of any importance at the division stage of the experiment. This is also the case if one only considers the influence of the procedural variables. Among the controls, only the age of a participant bears a marginal influence. This is, however, not a systematic influence, as the comparison across the three models shows.

Assigned procedure exerts a particularly strong influence. As Table 1 already made clear, the DC procedure is associated with much smaller payoffs than the two Knaster 
mechanisms. DC is thus not a particularly promising procedure. This makes it all the more puzzling that so many participants opted for this mechanism. The negative sign that the correspondence variable exhibits is particularly interesting. If a participant could not use the procedure for which she or he had originally opted, she or he can count on a higher payoff. This suggests that participants who were assigned to a procedure against their own preferences are bargaining more toughly at the division stage, trying more forcefully to maximize their payoffs than participants whose procedural preference was respected. Number of rounds is equally significant. The positive sign indicates that participants reached a higher payoff when they received in the beginning the message that they would only go through one division round. It is completely rational that individuals become greedier under a shortened time horizon.

Our results support in sum the thesis that psychological variables influence the choice of the fair-division procedure. Yet interestingly, these factors no longer have an impact once the institutional setting is given. Only procedural variables matter at this stage. This makes it mandatory that the inventors of a particular fair-division procedure care about the way in which their innovations are put into practical use. It is particularly important that a mechanism is not imposed against the will of the warring parties and that a sufficiently long time horizon is built up.

\section{CONCLUSION}

In an attempt to evaluate the empirical relevance of recently developed fair-division procedures, we found that these mathematical mechanisms perform well in a highly stylized experimental setting that imposes a strict bargaining protocol on participants. Yet we have to qualify this conclusion and therefore also the expectations of the proponents of fair-division procedures. The first and most important limitation is that the experimental versions of AK and PK only promise fairer solutions in contrast to a rough procedure such as DC in a world where actors are forced to adhere to the "rules of the game." If the bargaining protocol is not binding and incentives to deviate from the cooperative course start to lurk in the actors' minds, we see a considerable drop in the performance of the advanced procedures. Second, we can also observe that the choice of fair-division procedures has some important psychological foundations. This finding limits the usefulness of propagating mechanisms that are not compatible with the personality of a certain negotiator. Some negotiators might even opt for something as rough and inefficient as the DC procedure, although this mechanism promises a lower average payoff than the other procedures. This suggests that policy recommendations should not only account for the incentive structure but also for whether the proposed solutions are compatible with the psychological profile of the people involved. Although risk-acceptant people might find the mathematically sophisticated procedures more appealing, risk-averse individuals might prefer to opt for a straightforward and well-known mechanism such as DC. Third, the payoff a participant receives in the end depends very much how a procedure is implemented. If participants cannot divide the goods under a procedure of their own choice, they are more eager to maximize their payoff. A shortened time horizon is equally detrimental. 
Although some aspects remain necessarily unexplored, the work presented in this article is, to our knowledge, the first comparative empirical evaluation of fair-division procedures. It shares with other experiments the drawback of possible design effects. A critical assumption underlying the models and the tests presented in this article is that negotiators possess neither a common past nor a common future. This is in obvious contrast to most social situations where an agreement on a fair-division procedure would be most welcome: recurrent violent conflicts or separations that leave at least one side deeply frustrated. Because fair-division procedures see the bargaining process leading to the final settlement of a conflict as a single-shot interaction, the currently available mechanisms unfortunately do not hold large promise for these unhappy "relationships." Even if combatants or a couple agree to separate peacefully, the division on which they agree is, in real life, almost never complete and definitive. Separated territories continue to share borders, and divorcees remain often collectively responsible for the education of their children. Even if they initially considered a settlement fair, they might wish to reverse it once they have regained strength. Furthermore, it might be nearly impossible to convince warring parties in especially nasty conflicts to agree to a fair-division procedure in the first place. As the quantitative literature on conflict management shows, conflict dyads that agree on mediation are not a self-selected sample of all militarized interstate disputes (Schneider, Selck, and Bercovitch 2004). A final problem is that most actors possess relatively good knowledge of their partner's point allocation. Although they decide under uncertainty, their anticipatory strategic actions might prevent the conclusion of deals that both sides consider to be fair.

In short, the fair-division procedures we studied are most likely inadequate for the analysis of interactions between persons with a common future and past. We leave it to future work to identify fair-division mechanisms for these types of conflict. As our experimental evidence suggests, only procedures that match the psychological profile and the long-term incentives of the warring parties will ultimately offer convincing solutions to real-life conflicts.

\section{REFERENCES}

Bercovitch, Jacob, and Jeffrey Langley. 1993. The nature of the dispute and the effectiveness of international mediation. Journal of Conflict Resolution 37 (4): 670-91.

Brams, Steven J., and Alan D. Taylor. 1996. Fair division: From cake-cutting to dispute resolution. Cambridge: Cambridge University Press.

1999. The win-win solution: Guaranteeing fair shares to everybody. New York: Norton.

Diehl, Paul F., and Gary Goertz. 2000. War and peace in international rivalry. Ann Arbor: University of Michigan Press.

Edelman, Paul, and Peter Fishburn. 2001. Fair division of indivisible items among people with similar preferences. Mathematical Social Sciences 41:327-47.

Emery, Robert E. 1999. Marriage, divorce, and children's adjustment. Thousand Oaks, CA: Sage.

Farrell, Joseph, and Erik Maskin. 1989. Renegotiation in repeated games. Games and Economic Behavior 1:327-60.

Fehr, Ernst, and Klaus M. Schmidt. 1999. A theory of fairness, competition and cooperation. Quarterly Journal of Economics 114 (3): 817-68. 
Frohlich, Norman, and Joe A. Oppenheimer. 1992. Choosing justice: An experimental approach to ethical theory. Berkeley: University of California Press.

Frohlich, Norman, Joe A. Oppenheimer, and J. Bernard Moore. 2001. Some doubts about measuring selfinterest using dictator experiments: The costs of anonymity. Journal of Economic Behavior and Organization 46 (3): 271-90.

Hathaway, S. R., J. C. McKinley, and Rolf R. Engel, eds. 2000. MMPI-2. Minnesota Multiphasic Personality Inventory-2. Bern, Switzerland: Huber.

Knaster, Bronislaw. 1946. Sur le problème du partage pragmatique de H. Steinhaus. Annales de la Société Polonaise de Mathématique 19:228-30.

Krämer, Ulrike Sabrina, and Gerald Schneider. 2003. Faire Formeln. Psychologische und prozedurale Determinanten bei der Lösung von distributiven Konflikten. Kölner Zeitschrift für Soziologie und Sozialpsychologie 55:55-78.

Massoud, Tansa George. 2000. Fair division, adjusted winner procedure (AW), and the Israeli-Palestinian conflict. Journal of Conflict Resolution 44 (3): 333-58.

Michelbach, Philip A., John T. Scott, Richard E. Matland, and Brian H. Bornstein. 2003. Doing Rawls justice: An experimental study of income distribution norms. American Journal of Political Science 47 (3): 523-39.

Ostrom, Elinor, Roy Gardner, and James Walker 1994. Rules, games, and common-pool resources. Ann Arbor: University of Michigan Press.

Raith, Matthias. 2000. Fair-negotiation procedures. Mathematical Social Sciences 39:303-22.

Rawls, John. 1971/1999. A theory of justice. Rev. ed. Cambridge, MA: Harvard University Press.

Schneewind, Klaus A., and Johanna Graf. 1998. Der 16-Persönlichkeits-Faktoren-Test, Revidierte Fassung (16 PF-R). 5th ed. Bern, Switzerland: Huber.

Schneider, Gerald, Torsten Selck, and Jacob Bercovitch. 2004. The dispute or the mediator: The effectiveness of mediation in militarized disputes. Manuscript, University of Konstanz, Germany.

Sponsel, Rudolf. 1982. CST. Charakter-Struktur-Test. Erlangen, Germany: IEC.

Steinhaus, Hugo. 1948. The problem of fair division. Econometrica 16:101-4.

Uthmann, Jörg von. 1985. Die Diplomaten. Munich, Germany: DTV. 